\title{
SpinRCP: The Eclipse Rich Client Platform Integrated Development Environment for the Spin Model Checker
}

\author{
Zmago Brezočnik \\ Faculty of Electrical Engineering \\ and Computer Science \\ University of Maribor, Smetanova 17 \\ 2000 Maribor, Slovenia \\ +38622207210 \\ zmago.brezocnik@um.si
}

\author{
Boštjan Vlaovič \\ Faculty of Electrical Engineering \\ and Computer Science \\ University of Maribor, Smetanova 17 \\ 2000 Maribor, Slovenia \\ +38622207217 \\ bostjan.vlaovic@um.si
}

\author{
Aleksander Vreže \\ Faculty of Electrical Engineering \\ and Computer Science \\ University of Maribor, Smetanova 17 \\ 2000 Maribor, Slovenia \\ +38622207267 \\ aleksander.vreze@um.si
}

\begin{abstract}
SpinRCP is an integrated development environment for the Spin model checker used for verifying the correctnesses of concurrent and distributed systems. Using SpinRCP, it is easy to enter, edit, examine, and check the syntax of models which represent the systems to be analyzed, to check redundancies in models, to specify the required properties of models, to graphically represent processes and never claims derived from specified properties in the form of nondeterministic final state machines, to enter or select various simulation and verification parameters, to perform verification and random, guided, or interactive simulations and to transform a Spin simulation trail into a standard Message Sequence Chart (MSC). SpinRCP is implemented in Java as an Eclipse Rich Client Platform (RCP) product.
\end{abstract}

\section{Categories and Subject Descriptors}

D.2.4 [Software Engineering]: Software/Program Verification formal methods, model checking; D.2.6 [Software Engineering]: Programming Environments - integrated environments

\section{General Terms}

Verification

\section{Keywords}

Model checking, integrated development environment, Spin, SpinRCP, Eclipse Rich Client Platform

\section{INTRODUCTION}

The sizes and complexities of modern ICT systems and applications are increasing rapidly. On the other hand, designers are faced with demands for reductions in development costs and shorter time-to-market for new products. Additional difficulties for them stem from the fact that multithreaded, distributed, and/or parallel programs run concurrently. Fortunately, powerful formal

Permission to make digital or hard copies of all or part of this work for personal or classroom use is granted without fee provided that copies are not made or distributed for profit or commercial advantage and that copies bear this notice and the full citation on the first page. Copyrights for components of this work owned by others than ACM must be honored. Abstracting with credit is permitted. To copy otherwise, or republish, to post on servers or to redistribute to lists, requires prior specific permission and/or a fee. Request permissions from Permissions@acm.org.

SPIN'14, July 21-23, 2014, San Jose, CA, USA

Copyright 2014 ACM 978-1-4503-2452-6/14/07 ...\$15.00

http://dx.doi.org/10.1145/2632362.2632380 methods and tools are now available which can verify the logical correctness of concurrent software. Of one the more successful methods being used for the verification of real-life systems not only within academia but also in an industrial context is that of model checking. Perhaps the most widespread and useful model checking tool is Spin [5]. It is a command line tool. A user enters the commands at the command line and Spin outputs the results there. Such an approach to model checking can be difficult especially for newcomers who are not yet well-acquainted with Spin commands. Of course, the availability of a user-friendly graphical interface to Spin is of considerable benefit for skilled users as well. In order to accomplish these needs, several graphical interfaces/environments have been developed for Spin.

Xspin [5] was the first graphical interface for Spin. It was written in Tcl/Tk script language. The last version of Xspin was Version 5.2.5 from $17^{\text {th }}$ April 2010. Since then it has no longer been supported. jSpin [1] is an alternative graphical user interface for Spin. It was developed by M. Ben-Ari, together with its SpinSpider component, primarily for demonstrating the properties of concurrent programing. It is written in Java. Motivated by the need for a capable Promela editor when introducing our sdl2pml tool $[9,10]$ which generated a huge Promela model of an important part of the Iskratel SI3000 Softswitch specified in SDL, we developed an Eclipse Plug-in for Spin in Java [7]. iSpin [6] is the graphical user interface that has replaced Xspin since Spin Version 6.0.0. It has been provided by the Spin author, G. J. Holzmann, and is the default graphical user interface distributed with Spin. Just like Xspin, iSpin is implemented using the Tcl programing language and the Tk graphical user interface toolkit. EpiSpin [3], introduced by de Vos et. al, is another Eclipse plugin for editing Promela models and starting Spin verification and simulation runs. It is no longer supported. Still another tool called $\mathrm{COMPL}_{\mathrm{e}} \mathrm{T}_{\mathrm{e}}$ [4] is now used within the Eclipse. It is actually a toolchain for validating communication protocols which combines the possibility of an abstract behavior description represented as UML-Statechart models with a formal representation in Promela, which is used by Spin. It is not as yet publicly available. This paper introduces our new integrated development environment for Spin called SpinRCP $[8,2]$, which has superseded our previous tool. Comparisons regarding the basic facts about the existing Spin graphical user interfaces are given in Table 1.

The structure and main functionalities of the SpinRCP IDE are presented in Section 2. Section 3 provides information about the implementation details of the SpinRCP. Section 4 draws together its more important features and gives some ideas and plans for further work. 


\section{STRUCTURE AND MAIN FUNCTIONALITIES OF SPINRCP}

Based on the experiences gained in the development of Eclipse Plug-in for Spin [7], we decided to develop an integrated development environment for Spin users, which would facilitate editing and reviewing large Promela models including those extracted from an existing software code, simple parameters choosing for individual operations on the model, running Spin verification and simulation, graphical displays of MSCs, and keeping records of file versions. For the implementation of this environment, we selected the Eclipse Rich Client Platform (RCP) technology. RCP is the minimum set of plug-ins needed to build a rich client application. It allows us to quickly build a professionallooking application, with native look-and-feel, on multiple platforms. The application opens in a window called a Workbench that is entitled with SpinRCP and Spin versions and release dates, respectively. SpinRCP Workbench contains more perspectives. Each perspective contains parts (views and editors) and controls as to what appears in certain menus and tool bars. In SpinRCP, there are 19 different views (e.g. Model Navigator, Console, Simulation, Spin Trail to MSC, Help...) and only two editors (Promela Editor and MSC Viewer). Using a simple drag-and-drop operation you can relocate and/or resize any part, and thus reform the perspective at your will. Particular perspectives can be saved and later opened when needed. An outlook of SpinRCP Workbench perspective with the Menu Bar, the Tool Bar, the Model Navigator View, the Console View, the Promela Editor, the MSC Viewer Editor, the Simulation View, the Spin Trail to MSC View, and the CVS Repositories View, is shown in Figure 1.

SpinRCP needs four external tools: Spin, C compiler, Java, and Graphviz dot. The paths to these tools must be set on the Spin preference page. It is advised for ease of use to also set some useful options on the General preference page. Promela models can be either created or imported into the workspace within the Model Navigator View. Models in the workspace may be organized as tree-like folder/subfolder hierarchy. If you have an existing Promela model anywhere in your file system, you can simply copy-paste or drag-and-drop it to the appropriate place within the workspace. The following features are available for ease of viewing and editing models: syntax highlighting, code folding, content assist, and marking the place of a syntax error.

If a model in the Promela Editor is selected, several Tool Bar icons become enabled for launching a specific SpinRCP action. Syntax Check uses Spin -a option for performing a thorough model syntax check and generates the source $\mathrm{C}$ program for a model-specific verifier. If the Spin syntax checker detects a syntax error within the Promela source code, SpinRCP parses its error message and finds out the line number where the error occurred. The Promela Editor marks this line with an error icon.

Redundancy Check uses Spin -A option to apply a property-based slicing algorithm for the model, which can detect eventual redundancies in the model and generate suggestions on how the model could be revised in order to use less memory.

Symbol Table uses the Spin - $d$ option for producing symbol table information for the Promela model. The information for each Promela object depends on its type.

Automata View opens the Automata View preference page, where the user can select in which graphical format the automata should be displayed. Ten different file formats are currently available. SpinRCP uses options -03 and $-a$ to generate the verifier source $\mathrm{C}$ code, then compiles it to pan and runs pan using run-time option $-D$. This option generates state tables for each proctype and each never claim in the format accepted by the dot tool from Graphviz. These state tables are redirected to a text file. Next, the dot tool transforms this text file to a set of files (one file for automaton) with the previously selected graphical format. Then, a dialog appears, where the automaton to be displayed can be selected. Finally, a system program, assigned to a given file type is opened and the selected automaton is displayed.

The most powerful functionality of Spin that actually performs model checking is the verification. It is selected by clicking the Verification tab on the SpinRCP's Tool Bar. Now a quite complex Verification preference page is shown. In the upper part, the user can export current verification parameters (verification profile) to an $\mathrm{xml}$ file and import or reload a previously saved verification profile. Verification options are accessible below within two tabs: Basic Options and Advanced Options. In the Basic Options tab, a user can select a correctness property to be proved (either safety or liveness) with several additional options, the search mode, how

Table 1. Comparisons of basic facts about the existing Spin graphical user interfaces

\begin{tabular}{|l|l|l|l|l|l|}
\hline Software & Author & $\begin{array}{l}\text { Development } \\
\text { status }\end{array}$ & Latest version/Date & $\begin{array}{l}\text { Written in/ } \\
\text { Technology used }\end{array}$ & Download page/Website \\
\hline Xspin & Holzmann G.J. & $\begin{array}{l}\text { no longer } \\
\text { supported }\end{array}$ & $\begin{array}{l}\text { Version } 5.2 .5 \\
17 \text { April } 2010\end{array}$ & Tcl/Tk & - \\
\hline jSpin & Ben-Ari M. & $\begin{array}{l}\text { actively } \\
\text { developed }\end{array}$ & $\begin{array}{l}\text { Version } 5.0 \\
12 \text { December } 2010\end{array}$ & Java & https://code.google.com/p/jspin \\
\hline $\begin{array}{l}\text { Eclipse Plug- } \\
\text { in for Spin }\end{array}$ & Kovše T. et al. & $\begin{array}{l}\text { no longer } \\
\text { supported }\end{array}$ & $\begin{array}{l}\text { Version } 1.0 .4 \\
2 \text { May } 2011\end{array}$ & Java/Eclipse & http://lms.uni-mb.si/ep4s \\
\hline iSpin & Holzmann G.J. & $\begin{array}{l}\text { actively } \\
\text { developed }\end{array}$ & $\begin{array}{l}\text { Version } 1.1 .1 \\
22 \text { February } 2014\end{array}$ & Tcl/Tk & http://spinroot.com/spin/Src \\
\hline EpiSpin & De Vos B. et al. & $\begin{array}{l}\text { no longer } \\
\text { supported }\end{array}$ & $\begin{array}{l}\text { Version } 1.0 .4 \\
16 \text { November } 2011\end{array}$ & $\begin{array}{l}\text { Spoofax language } \\
\text { workbench }\end{array}$ & http://epispin.ewi.tudelft.nl \\
\hline COMPLeTe & Gröning S. et al. & not known & not known & Eclipse Plug-in & - \\
\hline SpinRCP & Brezočnik Z. et al. & $\begin{array}{l}\text { actively } \\
\text { developed }\end{array}$ & $\begin{array}{l}\text { Version 2.3.0 } \\
15 \text { June 2014 }\end{array}$ & $\begin{array}{l}\text { Java/Eclipse Rich } \\
\text { Client Platform }\end{array}$ & http://lms.uni-mb.si/spinrcp \\
\hline
\end{tabular}


a full queue behaves during verification (either blocks or loses new messages), the explicit use of user-entered compile-time and run-time parameters which supersede the clicked options and elsewhere entered parameters, and how a never claim (if any) is specified: with the in-model LTL formula/claim name (default), an LTL formula within the text field, an LTL formula in a 1-line file, and a never claim in a file. In the Advanced Options tab it is possible to enter several advanced verification parameters and select some error-trapping and verification run type options. If the verification finds an error, a counterexample is generated and saved in a so-called error-trail file with the file extension .trail appended to the original Promela model file name. The trail file can be used in guided simulation that replays the execution that violated the property.

Simulation opens the Simulation preference page, where the user can select the type of simulation (random, guided, interactive), the number of initial steps skipped, the maximum number of simulation steps, how a full queue is simulated (either blocks or loses new messages), a seed value for the random simulation, and the trail file for the guided simulation. Then the Simulation View opens and the simulation can start. Interactive simulation allows a user to select one from all executable Promela statements at a given moment in the interactive simulation dialog, in order to resolve the non-deterministic continuation of the execution run. Guided simulation uses an error-trail file produced during a verification run. Therefore, if the verification discloses an error, guided simulation will be used for displaying the execution run that violates the checked property of a model. Two Java threads run in parallel during the simulation: a Spin simulation thread and an MSC refreshing thread. The simulation thread executes the Spin simulation and displays Spin textual simulation outputs on the Console. In parallel, the MSC refreshing thread is displaying the MSC in the MSC Viewer. Currently, continuous and message- by-message simulations are available. The variable values' and queue contents' values are updated in two separate tables at the bottom of the Simulation View. In order to adapt the display of MSCs, the user can select or deselect particular messages in an MSC, rename selected messages, join processes into a new virtual process, and select whether to show or hide message parameters.

SpinRCP includes another view called Spin Trail to MSC which is intended for converting a Spin simulation output file (out) to the standard MSC text file (msc) formatted according to ITU-T Z.120, which can be displayed in graphical form using external design tools that support that standard. As for the viewing a MSC in the MSC Viewer, the same options are available to be set before the conversion. The abstraction using the introduction of virtual processes is very useful for analyzing huge MSCs. It hides details that are not interesting at a given abstraction level and would otherwise represent an unnecessary complexity.

The conversion can be done even more easily. If an MSC in the MSC Viewer is selected, the Export to MSC button in the Tool Bar is enabled. You just click it and enter or select the msc file name and the conversion begins.

The last button in the Tool Bar is the Import Trail button. When enabled, a click on this button opens a file dialog, where the out simulation trail file name to be read-in and displayed as a MSC in the MSC Viewer can be entered or selected. The same can be done by a double-click on an out file in the Model Navigator.

When analyzing a given model with Spin, it is often necessary to make several corrections of the model and/or the LTL formulas, to perform Spin actions with various parameters for simulation, verifier generation, compiling and running, to save simulation and verification outputs, MSC files etc. Therefore, it is very important to be able to cope with many versions of different files. This issue

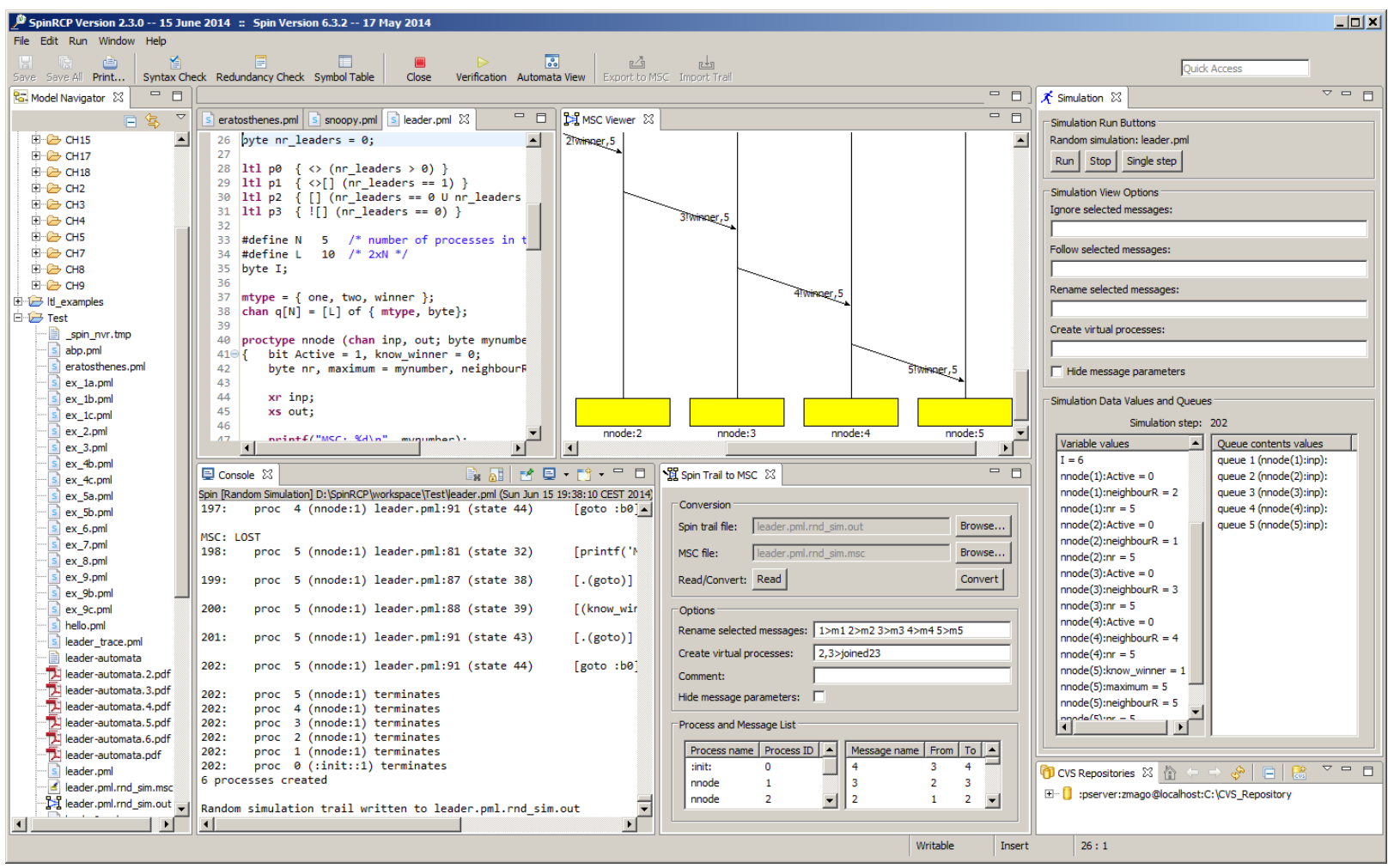

Figure 1. SpinRCP Workbench perspective with five different views and two editors 
is especially important in the case of team work. In the SpinRCP integrated development environment, the file version management is automatized using the CVS Repositories View. For example, a selected version of a model can be transferred from the local or remote CVS Repository into the SpinRCP environment, changed there, and stored back into the repository.

\section{IMPLEMENTATION DETAILS}

SpinRCP is written completely in Java within the Eclipse IDE (currently Eclipse Kepler Service Release 1) and then exported as an Eclipse Rich Client Platform (RCP) product. Therefore, it runs as a stand-alone RCP application without the need to have Eclipse IDE installed because all required plug-ins from Eclipse are already bundled in SpinRCP. The whole SpinRCP product consists of 122 plug-ins. One of them is our plug-in called org.um.feri.spin.rcp, which contains more than 16,000 lines of Java source code in 19 Java packages with a total of 84 files defining Java classes. The help contents for SpinRCP is implemented in a separate plug-in that contains more than $60 \mathrm{html}$ files with descriptions of individual help topics and many $\mathrm{xml}$ configuration files. In addition, SpinRCP uses a slightly modified version of the st2msc Java application [7] integrated as an internal jar file. SpinRCP is freely available at the following website: http://lms.uni-mb.si/spinrcp.

There you can find links to the required external software, download and installation instructions, SpinRCP release notes, a brief user guide through all functionalities, some references and answers to frequently asked questions. SpinRCP is available for the following platforms: Windows 32 bit (win32/x86), Windows 64 bit (win32/x86_64), Linux 32 bit (gtk/x86), Linux 64 bit (gtk/x86_64), Mac OS X 32 bit (cocoa/x86), and Mac OS X 64 bit (cocoa/x86 64). The SpinRCP installation for another (older) operating system (e.g. Solaris, HP UX, AIX) and/or hardware architecture can be generated on request. SpinRCP is compatible with all Spin versions, including those before Spin Version 6.0.0 that have different linenumber/filename references. Of course, some options might not work with older versions (e.g., the Automata View is feasible only from Spin Version 6.0.0).

The first publicly released version of SpinRCP was Version 2.2.0 on $28^{\text {th }}$ April 2014. Its latest version is 2.3.0 issued on $15^{\text {th }}$ June 2014. With the exception of iSpin, which is a default Spin graphical user interface, for no other known freely available Spin GUI a new version has been released in the last three years. On the other hand, SpinRCP has been actively developed further. New functionalities will be continuously added, the code improved and in particular it will offer support for the possible new features in upcoming distributions of Spin.

\section{CONCLUSION}

The more important features of SpinRCP are the following ones: a user-friendly Promela editor with syntax coloring, code folding, keyword autocompletion, and syntax error marking, running Spin verification, random, guided, and interactive simulation, graphical MSC viewing, abstracting MSCs by joining some processes into an abstract process, conversion of Spin simulation output to a standard text file, which is readable by external MSC viewers (e.g., ObjectGEODE), displaying graphical automata representation of proctype definitions and never claims in a model in ten different graphical formats. We have many ideas and plans for further improvements and new features of SpinRCP: options for filtering the Spin simulation output to console, a rewind option together with stepping forward and backwards during step simulation, indication of the statement that is currently executed during a simulation run in the Promela source file, cleanup of temporary files, display of a process creation in the MSC Viewer, a command for displaying the state tables for proctype definitions and never claims, an extension of the Automata View that displays the FSMs of proctype definitions and never claims of a corresponding Promela model by highlighting the visited states in the guided simulation run that illustrate the violation of the property being checked, Spin swarm support for distributing a model checking task to more CPU cores or to a cloud of workstations, a transition from the current CVS version control system to the more powerful GIT distributed revision control and source code management system, generation of verification reports in textual, tabular, and/or graphical form, etc. We hope for a positive response from the Spin model checking research and education community. Feedback information ever since the tool has been freely available is very promising.

\section{REFERENCES}

[1] Ben-Ari, M. 2010. jSpin - Java GUI for SPIN: User's Guide, Version 5.0. Department of Science Teaching, Weizmann Institute of Science, Rehovot, Israel.

[2] Brezočnik, Z., Vlaovič, B., and Vreže, A. 2013. Model Checking using Spin and SpinRCP. Inform. MIDEM. 43, 4(Dec. 2013), 235-250. (December 2013). Retrieved December 28, $2013 \mathrm{http}: / / \mathrm{www}$. midemdrustvo.si/Journal/Search.aspx? Vol=43\&Iss=4

[3] de Vos, B., Kats, L.C.L., Pronk, C. 2011. EpiSpin: An Eclipse Plug-In for Promela/Spin Using Spoofax, In: Groce, A., Musuvathi, M. (eds.) SPIN 2011. LNCS. 6823, 177-182. Springer, Heidelberg.

[4] Gröning, S., Rosas, C., Wietfeld, C. 2013. COMPL $\mathrm{C}_{\mathrm{e}}-\mathrm{A}$ COMmunication protocol validation Toolchain using Formal and Model-Based Specifications and Descriptions. In: Bartocci, E., Ramakrishnan C.R. (eds.): SPIN 2013. LNCS. 7976, 18-23. Springer, Heidelberg.

[5] Holzmann, G.J. 2003. The Spin Model Checker: Primer and Reference Manual. Addison-Wesley, Boston.

[6] Hornos, M.J., Augusto, J.C. 2012. Installation Process and Main Functionalities of the Spin Model Checker. (February 2012). Retrieved March 2, 2013 from http://digibug.ugr.es/handle/10481/19601

[7] Kovše, T., Vlaovič, B., Vreže, A., Brezočnik, Z. 2009. Eclipse plug-in for spin and st2msc tools - tool presentation. In: Păsăreanu, C.S. (ed.) SPIN 2009. LNCS. 5578, 143-147. Springer, Heidelberg.

[8] Kovše, T. 2011. Environment for formal verification of safety-critical systems, Master's Thesis (in Slovene), FERI, University of Maribor, Slovenia.

[9] Vlaovič, B., Vreže, A., Brezočnik, Z., Kapus, T. 2007. Automated generation of Promela model from SDL specification. Comp. Stand. Inter. 29, 4(May. 2007), 449-461. DOI=http://dx.doi.org/10.1016/j.csi.2006.10.001.

[10] Vreže, A., Vlaovič, B., Brezočnik, Z. 2009. Sdl2pml - tool for automated generation of Promela model from SDL specification. Comp. Stand. Inter. 31, 4(Jun. 2009), 779-786. DOI=http://dx.doi.org/10.1016/j.csi.2008.09.005. 